\title{
A Monotone Preservation Result for Boolean Queries Expressed as a Containment of Conjunctive Queries
}

\author{
Dimitri Surinx Jan Van den Bussche \\ Hasselt University
}

\begin{abstract}
When a relational database is queried, the result is normally a relation. Some queries, however, only require a yes/no answer; such queries are often called boolean queries. It is customary in database theory to express boolean queries by testing nonemptiness of query expressions. Another interesting way for expressing boolean queries are containment statements of the form $Q_{1} \subseteq Q_{2}$ where $Q_{1}$ and $Q_{2}$ are query expressions. Here, for any input instance $I$, the boolean query result is true if $Q_{1}(I)$ is a subset of $Q_{2}(I)$ and false otherwise.

In the present paper we will focus on nonemptiness and containment statements about conjunctive queries. The main goal is to investigate the monotone fragment of the containments of conjunctive queries. In particular, we show a preservation like result for this monotone fragment. That is, we show that, in expressive power, the monotone containments of conjunctive queries are exactly equal to conjunctive queries under nonemptiness.
\end{abstract}

\section{Introduction}

In this paper, we compare boolean queries (or integrity constraints) expressed using conjunctive queries (CQs [1]) in two different ways:

Nonemptiness: As an expression of the form $Q \neq \emptyset$, with $Q$ a CQ;

Containment: As an expression of the form $Q_{1} \subseteq Q_{2}$, with $Q_{1}$ and $Q_{2}$ two CQs.

An example of a nonemptiness query is "there exists a customer who bought a luxury product". An example of a containment query is "every customer who bought a luxury product also bought a sports product". A qualitative difference between nonemptiness and containment queries is that nonemptiness queries are always monotone: when the result is true on some input instance, it is also true on any larger instance. In contrasts, containment queries need not be 
monotone, as shown by the example above. The nonemptiness of a CQ is always expressible as the containment of two CQs. For example, the nonemptiness of $(x) \leftarrow$ Customer $(x)$, Bought $(x, y)$, Luxury $(y)$ is expressed as

$$
() \leftarrow \text { true } \subseteq \quad() \leftarrow \text { Customer }(x) \text {, Bought }(x, y), \operatorname{Luxury}(y) .
$$

Conversely, one may suspect that, as far as monotone queries are concerned, nothing more is expressible by a containment of two CQs. Indeed, we show in this paper that every monotone query expressed as the containment of two CQs is already expressible as the nonemptiness of a CQ. Such a result fits the profile of a preservation theorem since it gives a syntactical language for a semantical sublanguage. Preservation theorems have been studied intensively in model theory, finite model theory and database theory [7, 2, 6, 9, 8, 4].

From our proof it also follows that monotonicity testing of a containment of two CQs is decidable; specifically, the problem is NP-complete.

\section{Preliminaries}

A database schema $\Gamma$ is a finite nonempty set of relation names. Every relation name $R$ is assigned an arity, which is a natural number. Let $V$ be some fixed infinite universe of data elements and let $R$ be a relation name of arity $n$. An $R$-fact is an expression of the form $R\left(a_{1}, \ldots, a_{n}\right)$ where $a_{i} \in V$ for $i=1, \ldots, n$. Generally, a fact is an $R$-fact for some $R$. An $R$-instance $I$ is a finite set of $R$-facts. More generally, an instance $I$ of a database schema $\Gamma$ is defined to be a nonempty union $\bigcup_{R \in \Gamma} I(R)$, where $I(R)$ is an $R$-instance. The active domain of an instance $I$, denoted by $\operatorname{adom}(I)$, is the set of all data elements from $V$ that occur in $I$. An instance $I$ is called connected when for every two data elements $a, b \in \operatorname{adom}(I)$ there is a sequence of facts $f_{1}, \ldots, f_{n}$ in $I$ such that: $a$ is in $\operatorname{adom}\left(\left\{f_{1}\right\}\right), b$ is in $\operatorname{adom}\left(\left\{f_{n}\right\}\right)$, and $\operatorname{adom}\left(\left\{f_{i}\right\}\right) \cap \operatorname{adom}\left(\left\{f_{i+1}\right\}\right) \neq \emptyset$ for any $i=1, \ldots, n-1$. An instance $J$ is a called a connected component of $I$ if $J$ is connected, $J \subseteq I$ and $J$ is maximal in $I$ with respect to inclusion.

We have defined database and instances under the so called "logic programming perspective" [1. We will define the results of conjunctive queries, however, under the so-called "named" perspective [1. This will allow a lighter notation in our proof of Lemma 5 where we are taking subtuples of heads of conjunctive queries.

In the named perspective, tuples are defined over a finite set of attributes, which we refer to as a relation scheme. Formally, tuples, say $t=\left(u_{i}\right)_{i \in S}$ on a relation scheme $S$, are considered as mappings, so $t$ is a mapping on $S$ and $t(i)=u_{i}$. Then, subtuples, say $\left.t\right|_{K}$ for $K \subseteq S$ are treated as restrictions of the mapping $H$ to $K$. On the empty relation scheme, there is only one tuple, namely the empty mapping, also called the empty tuple. We denote the empty tuple by ().

We formalize the notion of conjunctive queries as follows. From the outset we assume an infinite universe of variables. A conjunctive query is an expression of the form $Q: H \leftarrow B$ where the head $H$ is a tuple of variables (tuple in the 
sense as just defined), and the body $B$ is a set of atoms over $\Gamma$. An atom is an expression of the form $R\left(v_{1}, \ldots, v_{n}\right)$ where $R \in \Gamma$ and $v_{1}, \ldots, v_{n}$ are variables. We will denote the set of conjunctive queries over $\Gamma$ as $\mathrm{CQ}_{\Gamma}$. For a conjunctive query $Q$ we will write $H_{Q}$ for the head and $B_{Q}$ for the body of $Q$. The result scheme of a conjunctive query $Q$ is the relation scheme of the head $H_{Q}$. Note that we allow unsafe queries, i.e., queries with head variables that do not appear in the body. Semantically, for any instance $I$ over $\Gamma, Q(I)$ is defined as:

$$
\left\{f \circ H_{Q} \mid f \text { is a homomorphism from } Q \text { into } I\right\} \text {. }
$$

Here, a homomorphism $f$ from $Q$ into $I$ is a function on the variables in $H_{Q}$ and $B_{Q}$ to adom $(I)$ such that $f\left(B_{Q}\right) \subseteq I$. When the variables in $H_{Q}$ are all present in $B_{Q}$, we will also write that $f$ is a homomorphism from $B_{Q}$ into $I$. Interchangeably, we will write that $B_{Q}$ maps into $I$.

Example 1. Consider the database schema with the relation name Flights of arity two. The following conjunctive query returns all the city pairs that are connected by flight with one stopover:

$$
(A: x, B: y) \leftarrow \operatorname{Flight}(x, z), \text { Flights }(z, y) .
$$

This query returns $\{(A:$ Vienna, $B:$ Brussels $),(A:$ Paris, $B:$ Rome $)\}$ on the instance

$$
\{\text { Flights(Paris, Brussels), Flights(Brussels, Rome), Flights(Vienna, Paris)\}. }
$$

Remark 2. It is convenient to assume that variables are data elements in $V$. Then, we can use the body of a conjunctive query as a database instance. As a consequence, an $R$-atom can then be thought of as an $R$-fact.

For any two queries $Q_{1}$ and $Q_{2}$, we write $Q_{1} \sqsubseteq Q_{2}$ if $Q_{1}(I) \subseteq Q_{2}(I)$ for any database instance $I$ over $\Gamma$. We recall:

Theorem 3 ([5]). Let $Q_{1}$ and $Q_{2}$ be conjunctive queries. Then, $Q_{1} \sqsubseteq Q_{2}$ iff $H_{Q_{1}} \in Q_{2}\left(B_{Q_{1}}\right)$.

A boolean query over a database schema $\Gamma$ is a mapping from instances of $\Gamma$ to $\{$ true, false $\}$. We can associate to any conjunctive query $Q$, a boolean query $Q \neq \emptyset$, that is true on $I$ if $Q(I) \neq \emptyset$ and false if $Q(I)=\emptyset$. We will write $\mathrm{CQ}_{\Gamma}^{\neq \emptyset}$ for the family of boolean queries of the form $Q \neq \emptyset$ where $Q$ is in $\mathrm{CQ}_{\Gamma}$.

As argued in the introduction, this is not the only natural way to express boolean queries. Containment statements of the form $Q_{1} \subseteq Q_{2}$ provide a clean way to express interesting nonmonotone boolean queries. Formally, the boolean query $Q_{1} \subseteq Q_{2}$ is true on $I$ if $Q_{1}(I)$ is a subset of $Q_{2}(I)$, and false on $I$ otherwise. It is understood that we can only take containment boolean queries of two conjunctive queries $Q_{1}$ and $Q_{2}$ if they have the same result scheme. We write $\mathrm{CQ} \stackrel{\subseteq}{\subseteq}$ for the family of boolean queries expressible by containment statements $Q_{1} \subseteq Q_{2}$ where $Q_{1}$ and $Q_{2}$ are in $\mathrm{CQ}_{\Gamma}$ with the same result scheme.

Recall that every conjunctive query $Q$ is monotone, in the sense that for any two instances $I, J$ over $\Gamma$, such that $I \subseteq J$, we have $Q(I) \subseteq Q(J)$. Furthermore, 
we say that a boolean query $Q$ is monotone if for any two instances $I, J$ over $\Gamma$, such that $I \subseteq J$, we have $Q(I)=$ true implies $Q(J)=$ true. We denote the set of monotone boolean queries with MON.

We will frequently use the following property of conjunctive queries with connected bodies. If $Q$ is a conjunctive query with a connected body, then $Q(I \cup J)=Q(I) \cup Q(J)$ for any domain-disjoint instances $I$ and $J$. We will refer to this property as the additivity property. Furthermore, we say that a query $Q$ is additive if it has the additivity property.

\section{Main result}

In this section we will prove the main theorem of the present paper. This preservation theorem can be summarized as follows:

Theorem 4. For any database schema $\Gamma, \mathrm{CQ}_{\Gamma}^{\subseteq} \cap \mathrm{MON}=\mathrm{CQ}_{\Gamma}^{\neq \emptyset}$. Specifically, every monotone query $Q_{1} \subseteq Q_{2}$, where $Q_{1}$ and $Q_{2}$ are $C Q s$, is equivalent to a query of the form $(() \leftarrow B) \neq \emptyset$, where $B$ is empty or $B$ consists of some of the connected components of $B_{Q_{2}}$.

Note that $\mathrm{CQ}_{\Gamma}^{\neq \emptyset} \subseteq \mathrm{CQ}_{\Gamma}^{\subseteq} \cap \mathrm{MON}$ already follows from the fact that $Q \neq \emptyset$ is equivalent to ()$\leftarrow \emptyset \subseteq() \leftarrow B_{Q}$. To prove the remaining inclusion we first establish a few technical results. First, we show that any monotone containment of conjunctive queries is equivalent to a containment of conjunctive queries with empty heads. For the remainder of this section, we write $Z_{a}$ to be the instance where there is exactly one fact $R(a, a, \ldots, a)$ for every $R \in \Gamma$. Note that for every CQ $Q$, we have $Q\left(Z_{a}\right)=\{(a, a, \ldots, a)\}$.

Lemma 5. Let $Q_{1}$ and $Q_{2}$ be conjunctive queries. If $Q_{1} \subseteq Q_{2}$ is monotone, then it is equivalent to the conjunctive query ()$\leftarrow B_{Q_{1}} \subseteq() \leftarrow B_{Q_{2}}$.

Proof. Let $S$ be the result scheme of $Q_{1}$ and $Q_{2}$. Write $B_{Q_{2}}$ as $B_{1}, \ldots, B_{k}, B$ where the $B_{j}$ are the connected components of $B_{Q_{2}}$ that contain at least one variable in $H_{Q_{2}}$, and $B$ is the collection of the remaining connected components.

Define $A_{j}=\left\{i \in S \mid H_{Q_{2}}(i) \in \operatorname{adom}\left(B_{j}\right)\right\}$ for $j=1, \ldots, k$ and let $A_{0}$ contain the remaining attributes in $S$. Furthermore, define $A=\bigcup_{1 \leq j \leq k} A_{j}$.

We first show that there is a function $f$ such that $\left.\left.f \circ H_{Q_{2}}\right|_{A_{0}} \stackrel{=H_{Q_{1}}}{=}\right|_{A_{0}}$. Let $a$ be a fresh data element. Define $I=Z_{a} \cup B_{Q_{1}} \cup \bigcup_{i \in C} Z_{H_{Q_{1}}(i)}$ where $C=\left\{i \in S \mid H_{Q_{1}}(i) \notin \operatorname{adom}\left(B_{Q_{1}}\right)\right\}$. Since, $Q_{1}\left(Z_{a}\right)=Q_{2}\left(Z_{a}\right)$ and $Q_{1} \subseteq Q_{2}$ is monotone, we have $Q_{1}(I) \subseteq Q_{2}(I)$. Therefore, $H_{Q_{1}} \in Q_{2}(I)$ since $H_{Q_{1}} \in Q_{1}(I)$. Hence, there is a homomorphism $f$ from $Q_{2}$ into $I$ such that $f \circ H_{Q_{2}}=H_{Q_{1}}$. In particular, $\left.f \circ H_{Q_{2}}\right|_{A_{0}}=\left.H_{Q_{1}}\right|_{A_{0}}$ as desired.

Next, we show for each $j=1, \ldots, k$ that

$$
\left(\left.H_{Q_{1}}\right|_{A_{j}} \leftarrow B_{Q_{1}}\right) \sqsubseteq\left(\left.H_{Q_{2}}\right|_{A_{j}} \leftarrow B_{j}\right) .
$$

Let $I$ be a nonempty instance over $\Gamma$ and let $a$ be a fresh data element. Suppose $t \in\left(\left.H_{Q_{1}}\right|_{A_{j}} \leftarrow B_{Q_{1}}\right)(I)$. Since $\left(\left.H_{Q_{1}}\right|_{A_{j}} \leftarrow B_{Q_{1}}\right)$ and $Q_{1}$ have the 
same body, and $\left.H_{Q_{1}}\right|_{A_{j}}$ is a subtuple of $H_{Q_{1}}$, we can extend $t$ to $t^{\prime}$ such that $t^{\prime} \in Q_{1}(I)$. Furthermore, since $Q_{1} \subseteq Q_{2}$ is monotone and $Q_{1}\left(Z_{a}\right)=Q_{2}\left(Z_{a}\right)$, we have $Q_{1}\left(I \cup Z_{a}\right) \subseteq Q_{2}\left(I \cup Z_{a}\right)$. Thus, $t^{\prime} \in Q_{2}\left(I \cup Z_{a}\right)$, whence we also have $t \in\left(\left.H_{Q_{2}}\right|_{A_{j}} \leftarrow B_{j}\right)\left(I \cup Z_{a}\right)$. Since $\left.H_{Q_{2}}\right|_{A_{j}} \leftarrow B_{j}$ is additive, $t \in\left(\left.H_{Q_{2}}\right|_{A_{j}} \leftarrow\right.$ $\left.B_{j}\right)(I) \cup\left(\left.H_{Q_{2}}\right|_{A_{j}} \leftarrow B_{j}\right)\left(Z_{a}\right)$. This implies that $t \in\left(\left.H_{Q_{2}}\right|_{A_{j}} \leftarrow B_{j}\right)(I)$ since $t$ is a tuple of data elements in $I$.

We now show that $Q_{1} \subseteq Q_{2}$ is equivalent to $Q_{1}^{\prime} \subseteq Q_{2}^{\prime}$ where $Q_{1}^{\prime}=() \leftarrow B_{Q_{1}}$ and $Q_{2}^{\prime}=() \leftarrow B_{Q_{2}}$, which proves our lemma. Clearly, $Q_{1}(I) \subseteq Q_{2}(I)$ implies that $Q_{1}^{\prime}(I) \subseteq Q_{2}^{\prime}(I)$. For the other direction, suppose that $Q_{1}^{\prime}(I) \subseteq Q_{2}^{\prime}(I)$ and let $t \in Q_{1}(I)$. Then, we have the following:

- There is a homomorphism $f_{1}$ from $B_{Q_{1}}$ to $I$ such that $f_{1} \circ H_{Q_{1}}=t$.

- There is a homomorphism $f_{2}$ from $B_{Q_{2}}$ to $I$ since $\emptyset \neq Q_{1}^{\prime}(I) \subseteq Q_{2}^{\prime}(I)$.

- There is a function $h$ such that $\left.h \circ H_{Q_{2}}\right|_{A_{0}}=\left.H_{Q_{1}}\right|_{A_{0}}$.

- For every $j=1, \ldots, k,\left.t\right|_{A_{j}} \in\left(\left.H_{Q_{2}}\right|_{A_{j}} \leftarrow B_{j}\right)(I)$ by $(\star)$. Hence, there is a homomorphism $h_{j}$ from $B_{j}$ into $I$ such that $\left.h_{j} \circ H_{Q_{2}}\right|_{A_{j}}=\left.t\right|_{A_{j}}$.

We now construct a homomorphism $f$ from $Q_{2}$ into $I$ such that $f \circ H_{Q_{2}}=t$. We define this $f$ as follows:

$$
f: x \mapsto \begin{cases}f_{2}(x), & \text { if } x \in B ; \\ h_{j}(x), & \text { if } x \in \operatorname{adom}\left(B_{j}\right) ; \\ f_{1} \circ h(x), & \text { otherwise. }\end{cases}
$$

We first show that $f \circ H_{Q_{2}}=t$.

$$
\begin{aligned}
f \circ H_{Q_{2}} & =f \circ\left(\left.\left.H_{Q_{2}}\right|_{A_{0}} \cup \bigcup_{1 \leq j \leq k} H_{Q_{2}}\right|_{A_{j}}\right) \\
& =\left.\left.f \circ H_{Q_{2}}\right|_{A_{0}} \cup \bigcup_{1 \leq j \leq k} f \circ H_{Q_{2}}\right|_{A_{j}} \\
& =\left.\left.f_{1} \circ h \circ H_{Q_{2}}\right|_{A_{0}} \cup \bigcup_{1 \leq j \leq k} h_{j} \circ H_{Q_{2}}\right|_{A_{j}} \\
& =\left.\left.f_{1} \circ H_{Q_{1}}\right|_{A_{0}} \cup \bigcup_{1 \leq j \leq k} t\right|_{A_{j}}=\left.\left.t\right|_{A_{0}} \cup \bigcup_{1 \leq j \leq k} t\right|_{A_{j}}=t
\end{aligned}
$$

Finally, we show that $f\left(B_{Q_{2}}\right) \subseteq I$.

$$
\begin{aligned}
f\left(B_{Q_{2}}\right)=f\left(B \cup \bigcup_{1 \leq j \leq k} B_{j}\right) & =f(B) \cup \bigcup_{1 \leq j \leq k} f\left(B_{j}\right) \\
& =f_{2}(B) \cup \bigcup_{1 \leq j \leq k} h_{j}\left(B_{j}\right) \subseteq I
\end{aligned}
$$


To prove Theorem 4 we may thus limit ourselves to conjunctive queries with empty heads. First, we have a look at containments of the form $Q_{1} \subseteq Q_{2}$ where $B_{Q_{1}}$ contains at least two non-redundant atoms. In what follows, when we write that a conjunctive query $Q$ is minimal, we mean that $B_{Q}$ does not contain redundant atoms. (An atom in $B_{Q}$ is called redundant if the query obtained from $Q$ by removing that atom is equivalent to $Q$.)

Lemma 6. Let $Q_{1}$ and $Q_{2}$ be CQs where $Q_{1}$ is minimal and $H_{Q_{1}}=H_{Q_{2}}=()$. If $B_{Q_{1}}$ contains at least two atoms, then $Q_{1} \subseteq Q_{2}$ is equivalent to true or is not monotone.

Proof. If $Q_{1} \subseteq Q_{2}$ is not equivalent to true, then $Q_{1} \nsubseteq Q_{2}$. Thus, $Q_{2}\left(B_{Q_{1}}\right)=\emptyset$ by Theorem [3, whence we have $Q_{1}\left(B_{Q_{1}}\right) \nsubseteq \subseteq Q_{2}\left(B_{Q_{1}}\right)$. Since $\left|B_{Q_{1}}\right| \geq 2$, there exists a nonempty $B \subsetneq B_{Q_{1}}$. We have $Q_{1}(B)=\emptyset$ for otherwise $Q_{1}$ would not be minimal.

Clearly, $Q_{1}(B)=\emptyset$ implies that $Q_{1}(B) \subseteq Q_{2}(B)$. Hence, $Q_{1} \subseteq Q_{2}$ is not monotone.

We are now ready to prove Theorem 4

Proof of Theorem 4. Let $Q_{1} \subseteq Q_{2}$ be in $\mathrm{CQ}_{\bar{\Gamma}}^{\subseteq} \cap \mathrm{MON}$. We want to show that $Q_{1} \subseteq Q_{2}$ is equivalent to $(() \leftarrow B) \neq \emptyset$ where $B$ is empty or $B$ consists of some of the connected components of $B_{Q_{2}}$.

By Lemma 5 we may assume that $H_{Q_{1}}=H_{Q_{2}}=()$. We may furthermore assume that $Q_{1}$ is minimal. The constant true query is expressed by ()$\leftarrow \emptyset \neq \emptyset$, so we may assume that $Q_{1} \nsubseteq Q_{2}$. Thus, $Q_{2}\left(B_{Q_{1}}\right)=\emptyset$ by Theorem 3 ,

If $B_{Q_{1}}$ contains at least two atoms, then $Q_{1} \subseteq Q_{2}$ is equivalent to true by Lemma 6 ,

If $B_{Q_{1}}=\emptyset$, then $Q_{1} \subseteq Q_{2}$ is equivalent to $Q_{2} \neq \emptyset$ which is in $\mathrm{CQ}_{\Gamma}^{\neq \emptyset}$.

Finally, suppose that $B_{Q_{1}}$ contains exactly one atom. First, let us consider $B_{Q_{1}}=\left\{R\left(x_{1}, \ldots, x_{n}\right)\right\}$ where there is a repetition among $x_{1}, \ldots, x_{n}$. Define $I_{1}=\left\{R\left(y_{1}, \ldots, y_{n}\right)\right\}$ where $y_{1}, \ldots, y_{n}$ are all different and not equal to any of $x_{1}, \ldots, x_{n}$. Clearly, $Q_{1}\left(I_{1}\right)=\emptyset$. Since $Q_{2}\left(B_{Q_{1}}\right)=\emptyset$, there is a connected component $C$ of $B_{Q_{2}}$ that does not map in $B_{Q_{1}}$. Furthermore, $C$ does not map into $I_{1}$ either, whence we also have $Q_{2}\left(I_{1}\right)=\emptyset$. Indeed, if $C$ would map into $I_{1}$, then $C$ would also map into $B_{Q_{1}}$ since $I_{1}$ maps into $B_{Q_{1}}$. It follows that $C$ does not map into $I_{1} \cup B_{Q_{1}}$ either, since $C$ is connected and adom $\left(I_{1}\right)$ is disjoint from $\operatorname{adom}\left(B_{Q_{1}}\right)$. Therefore, $Q_{2}\left(I_{1} \cup B_{Q_{1}}\right)=\emptyset$. Hence, $Q_{1}\left(I_{1} \cup B_{Q_{1}}\right) \nsubseteq Q_{2}\left(I_{1} \cup B_{Q_{1}}\right)$ since the head of $Q_{1}$ is in $Q_{1}\left(I_{1} \cup B_{Q_{1}}\right)$. This contradicts that $Q_{1} \subseteq Q_{2}$ is monotone, since $Q_{1}\left(I_{1}\right)=\emptyset \subseteq Q_{2}\left(I_{1}\right)$.

So, the only body left to consider is $B_{Q_{1}}=\left\{R\left(x_{1}, \ldots, x_{n}\right)\right\}$ where $x_{1}, \ldots, x_{n}$ are all different and $R \in \Gamma$. Our proof now depends on the size of $\Gamma$.

1. Suppose that $\Gamma$ only contains the relation name $R$. Then $Q_{1}(I) \neq \emptyset$ for any instance $I$ over $\Gamma$ since $B_{Q_{1}}=\left\{R\left(x_{1}, \ldots, x_{n}\right)\right\}$ where $x_{1}, \ldots, x_{n}$ are all different. Since $Q_{1}$ and $Q_{2}$ have empty heads, we may thus conclude that $Q_{1} \subseteq Q_{2}$ is equivalent to $Q_{2} \neq \emptyset$ in $\mathrm{CQ}_{\Gamma}^{\neq \emptyset}$. 
2. Suppose that $\Gamma$ only contains $R$ and exactly one other relation name $T$. Define $I_{1}=\left\{T\left(y_{1}, \ldots, y_{m}\right)\right\}$ where $y_{1}, \ldots, y_{m}$ are different from each other and from $x_{1}, \ldots, x_{n}$. Since the body of $Q_{1}$ is an $R$-atom and $I_{1}$ only contains a $T$-atom, we have $Q_{1}\left(I_{1}\right)=\emptyset$. Hence, $Q_{1}\left(I_{1}\right) \subseteq Q_{2}\left(I_{1}\right)$. By the monotonicity of $Q_{1} \subseteq Q_{2}$, we also have $Q_{1}\left(I_{1} \cup B_{Q_{1}}\right) \subseteq Q_{2}\left(I_{1} \cup B_{Q_{1}}\right)$. Therefore, every connected component of $B_{Q_{2}}$ maps in $I_{1}$ or $B_{Q_{1}}$. Indeed, $Q_{2}\left(I_{1} \cup B_{Q_{1}}\right) \neq \emptyset$ since the head of $Q_{1}$ is in $Q_{1}\left(I_{1} \cup B_{Q_{1}}\right)$. This observation partitions the connected components of $B_{Q_{2}}$ into two sets $B^{\prime}$ and $B^{\prime \prime}$, where $B^{\prime}$ contains the components that map into $I_{1}$, and $B^{\prime \prime}$ contains the components that map into $B_{Q_{1}}$.

We now show that $Q_{1} \subseteq Q_{2}$ is equivalent to $Q^{\prime}=() \leftarrow B^{\prime} \neq \emptyset$. To this end, suppose that $Q^{\prime}(I) \neq \emptyset$ and $Q_{1}(I) \neq \emptyset$ for some instance $I$ over $\Gamma$. Thus $B^{\prime}$ and $B_{Q_{1}}$ map into $I$. Since $B^{\prime \prime}$ maps into $B_{Q_{1}}$ by construction, we also have that $B^{\prime \prime}$ maps into $I$. Hence, $Q_{2}(I) \neq \emptyset$ as desired. For the other direction, suppose that $Q_{1}(I) \subseteq Q_{2}(I)$ for some instance $I$ over $\Gamma$. If $Q_{1}(I) \neq \emptyset$, then $Q_{2}(I) \neq \emptyset$ by assumption. Clearly, $Q^{\prime}(I) \neq \emptyset$ since $B_{Q^{\prime}}$ is a subset of $B_{Q_{2}}$. On the other hand, if $Q_{1}(I)=\emptyset$, then $I$ has no $R$-facts. Since instances cannot be empty, it must contain at least one $T$-fact, so $I_{1}$ maps into $I$. Thus $B^{\prime}$ also maps into $I$, whence $Q^{\prime}(I) \neq \emptyset$ as desired.

3. Finally, suppose that $\Gamma$ contains at least three relation names. Since $Q_{2}\left(B_{Q_{1}}\right)=\emptyset$, there is a connected component $C$ of $B_{Q_{2}}$ that does not map into $B_{Q_{1}}$. In particular, we know that $C$ is not empty, whence it contains at least one atom, say a $T$-atom. (Note that $T$ might be equal $R$.) Since there are three relation names in $\Gamma$ there is at least one other relation name $S$ in $\Gamma$ that is not equal to $T$ or $R$. Define $I_{2}=\left\{S\left(z_{1}, \ldots, z_{l}\right)\right\}$ where $z_{1}, \ldots, z_{l}$ are all different from each other and from $x_{1}, \ldots, x_{n}$. By construction, $C$ do not map into $I_{2}$ either, since $C$ contains an atom different from $S$. Thus, $Q_{2}\left(I_{2} \cup B_{Q_{1}}\right)=\emptyset$, whence we have $Q_{1}\left(I_{2} \cup B_{Q_{1}}\right) \not \subset Q_{2}\left(I_{2} \cup B_{Q_{1}}\right)$ since $Q_{1}\left(I_{2} \cup B_{Q_{1}}\right) \neq \emptyset$. However, $Q_{1}\left(I_{2}\right)=\emptyset$ since $R$ and $S$ are different, which implies that $Q_{1}\left(I_{2}\right) \subseteq Q_{2}\left(I_{2}\right)$. This contradicts the assumption that $Q_{1} \subseteq Q_{2}$ is monotone.

The proof of Theorem 4 gives us a procedure for deciding monotonicity for containments of CQs.

Corollary 7. Deciding whether a containment in $\mathrm{CQ}_{\Gamma}^{\subseteq}$ is monotone is NPcomplete.

Proof. Let $Q_{1} \subseteq Q_{2}$ be in $\mathrm{CQ}_{\bar{\Gamma}}$. By Lemma 5 we may remove the head variables of $Q_{1}$ and $Q_{2}$. The NP-hardness of our problem is taken care of by Lemma 6 . Indeed, when $B_{Q_{1}}$ contains at least two non-redundant body atoms, the problem is equivalent to deciding $Q_{1} \sqsubseteq Q_{2}$, which is known to be NP-hard [5].

Let us now show that the problem is in NP. By the proof of Theorem 4 we have the following cases when $Q_{1}$ is minimal: 
- If $B_{Q_{1}}=\emptyset$, then $Q_{1} \subseteq Q_{2}$ is always monotone.

- If $\left|B_{Q_{1}}\right| \geq 2$, then $Q_{1} \subseteq Q_{2}$ is monotone if and only if $Q_{1} \sqsubseteq Q_{2}$ (Lemma 6 ).

- If $Q_{1}=\left\{R\left(x_{1}, \ldots, x_{n}\right)\right\}$ where there is a repetition among $x_{1}, \ldots, x_{n}$, then $Q_{1} \subseteq Q_{2}$ is monotone if and only if $Q_{1} \sqsubseteq Q_{2}$.

- If $B_{Q_{1}}=\left\{R\left(x_{1}, \ldots, x_{n}\right)\right\}$ where $x_{1}, \ldots, x_{n}$ are all different, then:

(a) If $|\Gamma|=1$, then $Q_{1} \subseteq Q_{2}$ is always monotone;

(b) If $|\Gamma|=2$, then $Q_{1} \subseteq Q_{2}$ is always monotone;

(c) If $|\Gamma| \geq 3$, then $Q_{1} \subseteq Q_{2}$ is monotone if and only if $Q_{1} \sqsubseteq Q_{2}$.

These properties suggest the following algorithm:

1. Check if $B_{Q_{1}}=\emptyset$; if so, accept;

2. Check if $Q_{1} \sqsubseteq Q_{2}$; if so, accept;

3. Non-deterministically pick an atom $R\left(x_{1}, \ldots, x_{n}\right)$ in $B_{Q_{1}}$;

4. Check the following:

- ()$\leftarrow R\left(x_{1}, \ldots, x_{n}\right) \sqsubseteq Q_{1}$;

- $x_{1}, \ldots, x_{n}$ are all different.

5. Accept if $|\Gamma| \leq 2$ and the two checks above succeed; otherwise reject.

The containment checks (ㄷ) are well known to be in NP [5], so this algorithm is an NP algorithm.

If the algorithm accepts in step 1 , then $Q_{1} \subseteq Q_{2}$ is equivalent to $Q_{2} \neq \emptyset$, which is monotone. If the algorithm accepts in step 2, then the query $Q_{1} \subseteq Q_{2}$ is the constant true query, whence is trivially monotone. If the algorithm accepts in step 5 , then the query ()$\leftarrow R\left(x_{1}, \ldots, x_{n}\right)$ is equivalent to $Q_{1}$, which is clearly minimal. Hence, by cases $(a)$ and $(b)$ in the above properties, $Q_{1} \subseteq Q_{2}$ is monotone.

Conversely, suppose that $Q_{1} \subseteq Q_{2}$ is monotone. If $B_{Q_{1}}=\emptyset$ or $Q_{1} \sqsubseteq Q_{2}$, then the algorithm accepts in step 1 or 2 respectively. Otherwise, consider a CQ $Q_{1}^{\prime}$ obtained from $Q_{1}$ by omitting all redundant atoms. Certainly, $Q_{1}^{\prime}$ is minimal. Since $Q_{1}^{\prime} \subseteq Q_{2}$ is monotone and $Q_{1}^{\prime} \nsubseteq Q_{2}$, the above properties imply that $B_{Q_{1}^{\prime}}$ consists of a single atom $R\left(x_{1}, \ldots, x_{n}\right)$ where $x_{1}, \ldots, x_{n}$ are all different, and moreover that $|\Gamma| \leq 2$. Hence, by picking this atom in step 3 , the algorithm will accept. 


\section{Future Work}

There are several directions for future work. In this paper, conjunctive queries are not allowed to have constants in the head and/or body. Our proof method does not work in the presence of constants. Whether our characterization still holds in this case is still open.

Now that we have a syntactical characterization for monotone $\mathrm{CQ}^{\subseteq}$ we can look at other query languages. The first languages that come to mind are conjunctive queries with nonequalities, or negation, or unions. Another interesting language to consider is the more expressive first-order logic. When we allow infinite instances, the monotone first-order boolean queries are characterized by the positive first-order sentences with nonequalities 4. Whether this characterization still holds in restriction to finite instances remains open.

Another interesting line of work is to consider preservation theorems for other semantical properties, e.g., additivity. It can readily be verified that the additive queries in $\mathrm{CQ}^{\neq \emptyset}$ are exactly those with connected bodies. Another example of a preservation theorem for additivity is: connected Datalog $\urcorner$ captures the additive Datalog $\urcorner$ queries under stratified semantics [3].

\section{References}

[1] S. Abiteboul, R. Hull, and V. Vianu. Foundations of Databases. AddisonWesley, 1995.

[2] Miklos Ajtai and Yuri Gurevich. Monotone versus positive. J. ACM, 34(4):1004-1015, October 1987.

[3] Tom J. Ameloot, Bas Ketsman, Frank Neven, and Daniel Zinn. Datalog queries distributing over components. ACM Trans. Comput. Log., 18(1):5:1$5: 35,2017$.

[4] M. Benedikt, J. Leblay, B. ten Cate, and E. Tsamoura. Generating Plans from Proofs: The Interpolation-based Approach to Query Reformulation. Morgan\&Claypool, 2016.

[5] A.K. Chandra and P. Merlin. Optimal implementation of conjunctive queries in relational data bases. In Proceedings 9th ACM Symposium on the Theory of Computing, pages 77-90. ACM, 1977.

[6] C.C. Chang and H.J. Keisler. Model Theory. North-Holland, 3rd edition, 1990.

[7] Y. Gurevich. Toward logic tailored for computational complexity. In M.M. Richter et al., editors, Computation and Proof Theory, volume 1104 of Lecture Notes in Mathematics, pages 175-216. Springer-Verlag, 1984.

[8] Benjamin Rossman. Homomorphism preservation theorems. J. ACM, 55(3):15:1-15:53, August 2008. 
[9] Alexei P. Stolboushkin. Finitely monotone properties. In Proceedings of the 10th Annual IEEE Symposium on Logic in Computer Science, LICS '95, pages 324-, Washington, DC, USA, 1995. IEEE Computer Society. 